\title{
Disorders associated with abnormal acylcarnitine profile among high risk Egyptian children
}

\author{
El-Mesellamy $\mathrm{H}^{1}$, Gouda $\mathrm{AS}^{2}$, Fateen $\mathrm{E}^{2}$, Zaki $\mathrm{SS}^{2}$ \\ Biochemistry Department, Faculty of Pharmacy-Ain Shams University, Cairo, Egypt. \\ Amr_Gouda3@yahoo.com
}

\begin{abstract}
Acylcarnitine profile (ACP) is a useful tool in the biochemical diagnosis and monitoring of many acquired and inherited metabolic disorders. In the present study, acylcarnitines (ACs) were quantified in dried blood spot samples collected from 150 high risk Egyptian newborns and children using LC/MS/MS technique. They were referred to the Biochemical Genetics department in the National Research Center. Their age ranged from 1 to 36 months. Thirty seven patients had abnormal ACP diagnostic of some inherited metabolic disorders and other acquired conditions. The study revealed $5(13.5 \%)$ with medium chain acyl CoA dehydrogenase deficiency (MCADD), 1 (2.7 \%) with long chain hydroxyacyl CoA dehydrogenase deficiency (LCHADD), 1 (2.7 \%) with multiple acyl CoA dehydrogenase deficiency (MADD), 28 (75.7 \%) with secondary carnitine deficiency (SCD), 1 (2.7\%) with glutaric aciduria type I (GA I), and 1 (2.7\%) with methylmalonic aciduria (MMA) (Tab. 8, Ref. 39). Text in PDF www.elis.sk.

Key words: inborn errors of metabolism, fatty acid oxidation disorders, acylcarnitine profile, LC/MS/MS.
\end{abstract}

\begin{abstract}
Abbreviations: AC(s) - acylcarnitine(s), ACP - acylcarnitine profile, GA I - glutaric acidemia type I, LCHADD - long chain hydroxyacyl CoA dehydrogenase deficiency, LC/MS/MS - liquid chromatography mass-mass spectrometry, MADD - multiple acyl CoA dehydrogenase deficiency, MCADD - medium chain acyl CoA dehydrogenase deficiency, MMA - methylmalonic acidemia, MTP - mitochondrial trifunctional protein, OAP - organic acid profile, RI - renal insufficiency, SCD - secondary carnitine deficiency, VPA - valproic acid
\end{abstract}

The pathway of fatty acid oxidation contributes to energy homeostasis, especially in heart, liver and skeletal muscle. Acylcarnitines (ACs) are natural esters of fatty acids with free carnitine, which are formed during the process of fatty acid oxidation within the mitochondria. This oxidation process consists of many consecutive steps that are regulated by several enzymes, which are responsible for the estrification process and the transport of ACs through the mitochondrial membrane and another group of enzymes that regulate the $\beta$-oxidation process with subsequent energy production (1).

Deficiency or defect in one of these enzymes will lead to accumulation of ACs at the site of blockage (2). An increase or decrease in a single metabolite cannot be considered diagnostic for a particular metabolic disorder. The majority of metabolic disorders are represented with a characteristic pattern of excreted metabo-

${ }^{1}$ Biochemistry Department, Faculty of Pharmacy-Ain Shams University, Cairo, Egypt, and ${ }^{2}$ Biochemical Genetics Department, National Research Center, Cairo, Egypt

Address for correspondence: A.S. Gouda, Biochemical Genetics Department, National Research Center, Cairo, Egypt. lites. The confirmatory tests help to support the diagnostic value of the abnormal metabolic pattern. Hence, the abnormal presence of a single AC is not diagnostic of fatty acid oxidation disorder (FAOD); because an enzyme deficiency responsible for oxidation of a certain fatty acids group of the same chain length; will show an increase in almost all ACs within this group (3).

Analysis of ACP is complicated as the concentration of free carnitine and acylcarnitine varies with age where they decrease normally within the first week of life and dramatically increase in older infants making the interpretation of ACP more reliable (3). Another factor is that many metabolic disorders are accompanied by marked decrease in free carnitine with an elevation in many characteristic ACs. Also, the same consequences can be produced from chronic treatment with certain drugs (e.g. valproic acid, cisplatin, and cephalosporin) that cause lowering in free carnitine concentration by different mechanisms (3). The ACP is diagnostic for many inborn errors of metabolism (IEM) like:

FAODs that include:

I. Defects in carnitine cycle

II. Defects in the $\beta$-oxidation spiral

III. Genetic disorders of ketogenesis

Organic acid metabolism disorders (organic aciduria) (4).

The prognosis and monitoring the response to therapy in all these disorders can be achieved by the periodic ACP analysis. The application LC/MS/MS technique offers an accurate and a sensitive method for optimum detection of abnormal ACP (5).

Diagnosis of FAODs is performed principally by three methods. The first one is the measurement of the enzyme activity within the fibroblasts using labeled substrates (6). The second method is the molecular analysis of the mutations in the genes coding for the enzyme involved in the fatty acid oxidation pathway (6). These 
methods are relatively costly and time-consuming, whereas it is critical to get to a rapid diagnosis to manage the severe signs and symptoms of these disorders. The third method in FAODs diagnosis is the analysis of ACP using LC/MS/MS technique (or tandem mass spectrometry) (7).

\section{Subjects and methods}

\section{Subjects}

Dried blood spot, fresh urine, plasma and serum samples were obtained from 150 high risk children with age range from 1 to 36 months (with a mean of 16.2 months \pm 9.4 ) and 30 apparently healthy controls of matching age range (with a mean age of 14.7 months \pm 9.4$)$ were included. The study group included 78 (52 $\%)$ boys and $72(48 \%)$ girls. One hundred $(66.6 \%)$ of the high risk children were born to consanguineous parents. They were referred to the Biochemical Genetics department in the National Research Center (NRC) from different hospitals and clinics with suspicion of a metabolic disorder. A written consent was signed by the parents of the studied subjects and controls after full explanation of the study. This consent was obtained from the Ethical Committee at the NRC.

\section{Methods}

All suspected patients were subjected to the following investigations:

1) Measuring the levels of blood glucose, ammonia, lactate, blood glucose, transaminases, and total CK enzyme.

2) Qualitative detection of ketone bodies in urine.

3) Determining organic acid profile in urine (8).

4) ACP analysis in dried blood spot (5).

Chemicals: Stable isotopes internal standards (ISTDs) were obtained from Cambridge and included: ${ }^{2} \mathrm{H}_{9}$-carnitine (free carnitine, $\left.\mathrm{C}_{0}\right),{ }^{2} \mathrm{H}_{3}$-acetylcarnitine $\left(\mathrm{C}_{2}\right),{ }^{2} \mathrm{H}_{3}$-propionylcarnitine $\left(\mathrm{C}_{3}\right)$, ${ }^{2} \mathrm{H}_{3}$-butyrylcarnitine $\left(\mathrm{C}_{4}\right),{ }^{2} \mathrm{H}_{9}$-isovalerylcarnitine $\left(\mathrm{C}_{5}\right),{ }^{2} \mathrm{H}_{3}$-octanoylcrnitine $\left(\mathrm{C}_{8}\right),{ }^{2} \mathrm{H}_{9}$-myristolcarnitine $\left(\mathrm{C}_{14}\right)$, and ${ }^{2} \mathrm{H}_{3}$-palmitoylcarnitine $\left(\mathrm{C}_{16}\right)$. Unlabeled standards were obtained from Sigma and included: L-carnitine, acetyl-DL-carnitine, DL-hexanoylcarnitine, DL-myristoylcarnitine, DL-decanoylcarnitine, DL-laurylcarnitine and palmitoyl-L-carnitine. Methanol and acetonitrile HPLC grade from Sigma. Butanol-HCl (3M) from Sigma.

\section{Equipment}

The HPLC-ESI-MS system consisted of an HP 1100 Series HPLC instrument (quaternary pump and degasser, column compartment, and autosampler) and an LCQ Advantage MAX mass spectrometer from Thermo Finnigan. 'Xcalibure 1.4" software calculates the analyte concentration based on the deuterated ISTD. Two chromatographic eluents were used (relative volumes): eluent (A) acetonitrile (80\%) and eluent (B) $0.1 \%$ formic acid (20\%).

Preparation of AC butylesters from dried blood spots: Specimens were collected on to S\&S (Schleicher and Schuell). A single dried blood spot punch $3.2 \mathrm{~mm}$ diameter was placed in a polypropylene micro titer plate. Methanolic internal standard solution (100 ul) was added. The concentrations of the deuterium-labeled internal standards per liter of methanol were as follows: ${ }^{2} \mathrm{H}_{9}$-carnitine (free carnitine, $\left.\mathrm{C}_{0}\right) 0.76 \mathrm{nmol} / \mathrm{ml},{ }^{2} \mathrm{H}_{3}$-acetylcarnitine $\left(\mathrm{C}_{2}\right) 0.19 \mathrm{nmol} /$ $\mathrm{ml},{ }^{2} \mathrm{H}_{3}$-propionylcarnitine $\left(\mathrm{C}_{3}\right) 0.04 \mathrm{nmol} / \mathrm{ml},{ }^{2} \mathrm{H}_{3}$-butyrylcarnitine $\left(\mathrm{C}_{4}\right) 0.04 \mathrm{nmol} / \mathrm{ml},{ }^{2} \mathrm{H}_{9}$-isovalerylcarnitine $\left(\mathrm{C}_{5}\right) 0.04 \mathrm{nmol} / \mathrm{ml},{ }^{2} \mathrm{H}_{3}$ octanoylcrnitine $\left(\mathrm{C}_{8}\right) 0.04 \mathrm{nmol} / \mathrm{ml},{ }^{2} \mathrm{H}_{9}$-myristolcarnitine $\left(\mathrm{C}_{14}\right)$ $0.04 \mathrm{nmol} / \mathrm{ml}$, and ${ }^{2} \mathrm{H}_{3}$-palmitoylcarnitine $\left(\mathrm{C}_{16}\right) 0.08 \mathrm{nmol} / \mathrm{ml}$. The microtiter plate was gently shaken for 20 minutes. The methanol extract was transferred to a second microtiter plate and dried by a hot air blower. Butanol-HCl (70ul) was added to each sample well and the microtiter plate was covered with a thin Teflon sheet under a heavy weight and placed in $65^{\circ} \mathrm{C}$ for 15 minutes. After the plate was removed from the oven, the butanol- $\mathrm{HCl}$ was removed by a hot air blower. The butanol-derivatized samples were then reconstituted with (100ul) of the mobile phase (80:20 A: B by volume) and the plate was then covered with aluminum foil. The samples were then ready for MS/MS analysis.

Electrospray ionization mass- mass spectrometry (ESI MS/ MS) analysis: For MS/MS based testing, AC butylesters were analyzed without chromatographic separation in the positive ion mode, using precursor-ion (parent ion) scan. In the precursor ion mode for the analysis of AC butyl esters, all precursors of products $\mathrm{m} / \mathrm{z} 85$ within a mass range between 200 and $500 \mathrm{~m} / \mathrm{z}$ were recorded. The collision energy used was $35 \%$.

\section{Tuning method}

Source voltage; $4.5 \mathrm{KV}$, source current; $100 \mathrm{uA}$, capillary voltage; $3 \mathrm{~V}$, capillary temperature; $200{ }^{\circ} \mathrm{C}$, sheath gas flow; 60 arb., Helium was used as a collision gas at a pressure of 2.5 to 3.5 mTorr, the collision energy was set to $25 \mathrm{eV}$, tube lens offset; $5 \mathrm{~V}$, octapole RF amplifier $500 \mathrm{Vp}$-p, octapole offset (1); -2.5 V, octapole offset (2); $7 \mathrm{~V}$, entrance lens; $-20 \mathrm{~V}$, and interoctapole lens voltage; $-40 \mathrm{~V}$.

For the experiment involved internal standard, the ratio of the peak area of the AC in the sample to the peak area of the internal standard is calculated to provide an area ratio. When peak area ratios of several known external standards are measured, a plot of concentration versus area ratio can be constructed to provide a calibration curve. The quantitative analysis is accomplished by comparing the measured peak area ratio of a sample to the calibration curve and reading the concentration of the AC (according to the curve) that gave rise to that that peak area ratio. This is the calculated amount.

All the statistical analysis was performed using Statistical Package for Social Science (SPSS) version 9 software package, USA.

\section{Results}

The study group included $16(10.6 \%)$ high risk children with history of sibling deaths. Causes of death varied between cardiac arrest, or respiratory failure, or neurologic complications, or unexplained death.

The total high risk children were classified according to clinical presentation into acute and chronic presentation (Tab. 1).

The clinical manifestations in the study group were classified 
Tab. 1. Clinical presentation in the study group.

\begin{tabular}{lcc}
\hline $\begin{array}{l}\text { Clinical } \\
\text { presentation }\end{array}$ & $\begin{array}{c}\text { Acute condition } \\
\text { (metabolic crisis) }\end{array}$ & $\begin{array}{c}\text { Chronic conditions } \\
\text { (recurrent metabolic episodes) }\end{array}$ \\
\hline $\begin{array}{l}\text { No. of study group } \\
\text { (from total 150) }\end{array}$ & $109(72.6 \%)$ & $41(27.3 \%)$ \\
\hline
\end{tabular}

into neurologic, cardiac, hepatic, and muscular manifestations in addition to other manifestations (Tab. 2).

Measurement of blood glucose level and detecting ketone bodies in the urine of high risk children was performed to determine the presence or absence of non ketotic hypoglycemia (Tab. 3).

Levels of biochemical markers (blood glucose, $\mathrm{NH}_{3}$, lactate, transaminases, and total CK enzyme) of the high risk patients, values are expressed as the mean $\pm \mathrm{SD}$ are shown in Table 4 .

Distribution of the study group according to their ACP and the corresponding urinary organic acid profile is shown in Table 5.

From the previous results, it was emphasized that there were 37 patients had abnormal ACP among the study group; 5 patients with medium chain acyl CoA dehydrogenase deficiency (MCADD), 1 patient with 3-hydroxy long chain acyl CoA dehydrogenase deficiency (LCHADD), 1 patient with multiple acyl CoA dehydrogenases deficiency (MADD), and 28 patients with secondary carnitine deficiency (SCD) (3 patients with previously diagnosed renal insufficiency (RI), and 25 patients on chronic valproic acid (VPA) therapy), in addition to, 2 patients with other metabolic disorders (1 patient with glutaric aciduria type I (GA I), and 1 patient with methylmalonic aciduria (MMA)). Seven patients $(18.9 \%)$ had acute presentation and $30(81 \%)$ patients had chronic presentation. The clinical manifestations of these patients are shown in the Table 6 .

The presence of non ketotic hypoglycemia in diagnosed patients is shown in Table 7.

Biochemical markers (blood glucose, $\mathrm{NH}_{3}$, lactate, transaminases, and total CK enzyme) of diagnosed patients are shown in Table 8; values are expressed as the mean $\pm \mathrm{SD}$.

LC/MS/MS analysis of ACP:

ACs in dried blood spot after being derivatized into butyl ester derivatives, were ready for undergoing mass spectrometric analysis.

The fragmentation process starts with the removal of the trimethylammonium ion $\left(\mathrm{CH}_{3}\right)_{3} \mathrm{~N}^{+}$and the butyl group added during the derivatization process $\left(\mathrm{C}_{4} \mathrm{H}_{8}\right)$, thereby, decreasing the mass by $\mathrm{m} / \mathrm{z} 115$, then the removal of the fatty acid group $\left(\mathrm{CH}_{3}\left(\mathrm{CH}_{2}\right)\right.$ ${ }_{n} \mathrm{COOH}$ ), decreasing the mass by $\mathrm{m} / \mathrm{z}$ equal to the mass of the removed fatty acid group until reach the final fragment and the end fragment in the mass fragmentation of all AC species, $\mathrm{m} / \mathrm{z} 85$ $\left({ }^{+} \mathrm{CH}_{2} \mathrm{CH}=\mathrm{CHCOOH}\right)$.

The ACP analysis of the MCADD-patients revealed elevated concentrations of medium chain ACs; $\left(\mathrm{C}_{6}, \mathrm{C}_{8}, \mathrm{C}_{10}, \mathrm{C}_{10: 1}\right.$ and $\mathrm{C}_{8} /$
Tab. 3. Number and Percentage of high risk patients had non ketotic-hypoglycemia.

\begin{tabular}{lcc}
\hline Parameters & \multicolumn{2}{c}{ High risk children } \\
\cline { 2 - 3 } & No. from total 150 & Percent \\
\hline Hypoglycemia & 29 & $19.3 \%$ \\
Non ketotic urine & 108 & $72 \%$ \\
Non ketotic-hypoglycemia & 13 & $8.7 \%$ \\
\hline
\end{tabular}

Tab. 4. Mean \pm SD of measured biochemical markers in the controls and the high risk children.

\begin{tabular}{lcc}
\hline Biochemical markers & Controls & High risk children \\
\hline Blood glucose $(\mathrm{mg} / \mathrm{dl})$ & $91 \pm 8.7$ & $73.8 \pm 15.9$ \\
$\mathrm{NH}_{3}(\mu \mathrm{mol} / \mathrm{l}) \mathrm{n}=62$ & $6.1( \pm 2.5)$ & $62.8( \pm 54.2)$ \\
Lactate $(\mathrm{mg} / \mathrm{dl}) \mathrm{n}=24$ & $3.9( \pm 1.6)$ & $8.6( \pm 6.2)$ \\
Transaminases $(\mathrm{U} / \mathrm{L}) \mathrm{n}=78$ & & \\
$\quad \mathrm{ALT}$ & $21.66( \pm 5.31)$ & $339.6( \pm 856)$ \\
$\mathrm{AST}$ & $29.96( \pm 5.9)$ & $346.3( \pm 924)$ \\
$\mathrm{CK}(\mathrm{U} / \mathrm{L}) \mathrm{n}=23$ & $35.56( \pm 12.7)$ & $385.7( \pm 1605)$ \\
\hline
\end{tabular}

" $\mathrm{n}=$ No. of high risk children with elevated biochemical markers.

The difference in levels of all the biochemical markers between the controls and the study group was significant $(\mathrm{p}<0.001)$.

$/ \mathrm{C}_{10}$ ). They were detected at masses $\mathrm{m} / \mathrm{z} 316, \mathrm{~m} / \mathrm{z} 344, \mathrm{~m} / \mathrm{z} 372$, and $\mathrm{m} / \mathrm{z} 370$ respectively.

The ACP analysis showed increased amounts of 3-hydroxylong chain ACs. $\mathrm{C}_{14}-\mathrm{OH}$ was detected at $\mathrm{m} / \mathrm{z} 442, \mathrm{C}_{16}-\mathrm{OH}$ at $\mathrm{m} / \mathrm{z} 472$, $\mathrm{C}_{18}-\mathrm{OH}$ at $\mathrm{m} / \mathrm{z} 500$, and $\mathrm{C}_{18: 1}-\mathrm{OH}$ at $\mathrm{m} / \mathrm{z} 489$. Long chain $\mathrm{ACs}$ also were mild elevated like $\mathrm{C}_{16}, \mathrm{C}_{18}$, and $\mathrm{C}_{18: 1}$ and they were detected at masses $\mathrm{m} / \mathrm{z} 456, \mathrm{~m} / \mathrm{z} 484$, and $\mathrm{m} / \mathrm{z} 482$ respectively.

The ACP of MADD-patient showed an elevation in most $\operatorname{ACs}\left(\mathrm{C}_{4}-\mathrm{C}_{18}\right)$.

For RI-patients undergoing hemodialysis therapy; before the hemodialysis session; there was an abnormal increase in concentrations of both $\mathrm{C}_{0}$ and $\mathrm{C}_{2}$ detected at $\mathrm{m} / \mathrm{z} 218$ and 260 respectively, whereas after termination of the hemodialysis; analysis of the ACP of the same patients indicated that there was a marked decrease in $\mathrm{C}_{0}$ concentration.

For patients were on chronic VPA therapy; their ACP showed high concentrations of $\mathrm{C}_{3}$ and $\mathrm{C}_{5}$ detected at masses $\mathrm{m} / \mathrm{z} 274$ and $\mathrm{m} / \mathrm{z} 302$ respectively.

For GA I patient; ACP analysis revealed elevated amounts of $\mathrm{C}_{5} \mathrm{DC}$ at $\mathrm{m} / \mathrm{z} 388$ and $\mathrm{C}_{4}$ at $\mathrm{m} / \mathrm{z} 288$.

For MMA patient; ACP analysis showed elevated $\mathrm{C}_{4}$ at $\mathrm{m} / \mathrm{z}$ $288, \mathrm{C}_{3}$ at $\mathrm{m} / \mathrm{z} \mathrm{274}$, and $3-\mathrm{OHC}_{4}$ at $\mathrm{m} / \mathrm{z} 304$, and decreased $\mathrm{C}_{0}$.

\section{Discussion}

The FAODs are mainly concerned with an insufficient energy production as a result of decreased ketogenesis due to the limited production of acetyl CoA especially in status of fasting (9).

Tab. 2. Clinical manifestations in the study group.

\begin{tabular}{lccccc}
\hline $\begin{array}{l}\text { No. of high risk children } \\
\text { from total } 150\end{array}$ & \multicolumn{3}{c}{ Clinical manifestations } \\
\cline { 2 - 6 } & Neurologic & Cardiac & Hepatic & $\begin{array}{c}\text { Muscular } \\
\text { Gastric }\end{array}$ & $\begin{array}{c}\text { Respiratory } \\
\text { Other characteristic } \\
\text { manifestations }\end{array}$ \\
\hline $\begin{array}{l}\text { Number } \\
\text { Percent }\end{array}$ & 94 & 20 & 80 & 69 & 9 \\
\hline
\end{tabular}


Tab. 5. ACP and organic acids profile among the study group.

\begin{tabular}{|c|c|c|c|}
\hline \multicolumn{2}{|c|}{$\begin{array}{c}\text { The study group } \\
\text { (total 150) }\end{array}$} & \multirow[t]{2}{*}{$\mathrm{ACP}$} & \multirow[t]{2}{*}{ Urine organic acid profile } \\
\hline Number & Percent & & \\
\hline 113 & $75.3 \%$ & Normal profile & Normal profile \\
\hline 5 & $3.33 \%$ & $\uparrow$ Medium chain ACs & Medium chain dicarboxylic acids \\
\hline 1 & $0.66 \%$ & $\uparrow$ Long chain 3-hdroxy ACs & Long chain 3-hdroxy dicarboxylic acids \\
\hline 1 & $0.66 \%$ & $\uparrow$ Short-, medium-, long chain ACs & Glutaric, ethylmalonic\& short-, medium-, long chain dicarboxylic acids \\
\hline $3 *$ & $2 \%$ & $\uparrow$ Free carnitine\& acetylcarnitine & Normal profile \\
\hline $3 *$ & $2 \%$ & $\downarrow$ Free carnitine & Normal profile \\
\hline 25 & $16.7 \%$ & $\uparrow$ Propionyl-\& pentanoylcarnitine $\downarrow$ Free carnitine & Normal profile \\
\hline 1 & $0.66 \%$ & $\uparrow$ Glutaryl-\& butyrylcarnitine $\downarrow$ Free carnitine & Glutaric \& 3-OH glutaric \\
\hline 1 & $0.66 \%$ & $\begin{array}{l}\uparrow \text { Propionyl-, butyryl-\& hydroxy butyrylcarnitine } \\
\downarrow \text { Free carnitine }\end{array}$ & Methylmalonic, Methylcitric\& 3-OHpropionic acid \\
\hline
\end{tabular}

N.B. $(*)$ : The same patients

Tab. 6. No. of diagnosed patients in relation with the presented clinical manifestations.

\begin{tabular}{lccccccl}
\hline \multirow{2}{*}{ Group } & \multicolumn{6}{c}{ No. of patients had the clinical manifestations } \\
\cline { 2 - 8 } & $\begin{array}{c}\text { Neurologic } \\
\text { manifestations }\end{array}$ & $\begin{array}{c}\text { Cardiac } \\
\text { manifestations }\end{array}$ & $\begin{array}{c}\text { Hepatic } \\
\text { manifestations }\end{array}$ & $\begin{array}{c}\text { Muscular } \\
\text { manifestations }\end{array}$ & Vomiting & $\begin{array}{c}\text { Respiratory } \\
\text { manifestation }\end{array}$ & $\begin{array}{l}\text { Other characteristic } \\
\text { manifestations }\end{array}$ \\
\hline MCADD & - & 1 & 4 & - & 3 & 2 & - \\
\hline LCHADD & 1 & - & - & 1 & - & - & retinopathy \\
\hline MADD & 1 & 1 & 1 & 1 & - & - & Facial malformation, cystic renal disease. \\
\hline SCD & 15 & 16 & 13 & 15 & - & - & - \\
- RI & - & 3 & - & 3 & - & - & \\
- VPA & 15 & 13 & 13 & 12 & - & - & - \\
\hline Other metabolic diseases & 2 & 1 & - & - & - & - & Macrocephaly\& movement disorders. \\
GAI & 1 & - & - & - & - & - & Osteoporosis \\
MMA & 1 & - & - & - & - & - & 2 \\
\hline Total & 19 & 19 & 18 & 17 & 3 & 2 & 2 \\
\hline
\end{tabular}

N.B. No. of diagnosed patients related to the presented clinical manifestations is $>$ the total No. of the diagnosed patients as one patient might have more than one clinical manifestation.

Tab. 7. Blood glucose state, ketone bodies in urine, and presence or absence of non ketotic-hypoglycemia in the diagnosed patients.

\begin{tabular}{|c|c|c|c|c|c|c|c|}
\hline$\underline{\text { Parameter }}$ & MCADD & LCHADD & MADD & RI & VPA & GA I & MMA \\
\hline Blood glucose state & Normal or borderline & Low & Low & Low & Low & Low & Low \\
\hline Ketone bodies in urine & Hypo-ketonuria & Non ketotic urine & Non ketotic urine & Non ketotic urine & Non ketotic urine & ketonuria & ketonuria \\
\hline Non ketotic hypoglycemia & Absent & Present & Present & Present & Present & Absent & Absent \\
\hline
\end{tabular}

Tab. 8. Measured biochemical markers in the diagnosed patients.

\begin{tabular}{|c|c|c|c|c|c|c|c|}
\hline \multirow[b]{2}{*}{ Biochemical marker } & \multicolumn{7}{|c|}{ Disorder } \\
\hline & $\begin{array}{c}\mathrm{MCADD} \\
(\mathrm{mean} \pm \mathrm{SD})\end{array}$ & LCHADD & MADD & $\begin{array}{c}\mathrm{RI} \\
(\mathrm{mean} \pm \mathrm{SD})\end{array}$ & $\begin{array}{c}\mathrm{VPA} \\
(\mathrm{mean} \pm \mathrm{SD})\end{array}$ & GA I & MMA \\
\hline Blood glucose (mg/dl) & $69.2 \pm 4.2$ & 52 & 53 & $72.3 \pm 9.07$ & $74.7 \pm 11.2$ & 54 & 51 \\
\hline $\mathrm{NH} 3(\mu \mathrm{mol} / \mathrm{l})$ & $35 \pm 27.6$ & 11 & 197 & $58 \pm 18.4$ & $152 \pm 46.2$ & 37 & 97 \\
\hline Lactate $(\mathrm{mg} / \mathrm{dl})$ & $18 \pm 2.5$ & 34 & 29 & $22.7 \pm 4.5$ & $12 \pm 6$ & 18 & 22 \\
\hline \multicolumn{8}{|l|}{ Transaminases (U/L) } \\
\hline ALT & $647.6 \pm 247$ & 52 & 145 & $67.7 \pm 6.5$ & $1632 \pm 1559$ & 90 & 34 \\
\hline AST & $924 \pm 569.8$ & 19 & 187 & $63 \pm 11.5$ & $1637 \pm 1785$ & 110 & 42 \\
\hline CK (U/L) & $51.6 \pm 21.5$ & 18070 & 1982 & $1431 \pm 116.5$ & $351 \pm 668.4$ & 385 & 185 \\
\hline
\end{tabular}

The various manifestations involved in FAODs are due to affection of many organs in the body, together with the broad range of age; make this class of IEM very difficult to diagnose (10).

As the consanguineous marriages are common in Egypt; the probability of having an inherited autosomal recessive IEM must be considered. Temtamy et al (11) stated that the rate of consanguineous marriages among general population in Egypt was $20-40 \%$.
The low production of ketone bodies is concomitant with the depletion of glucose stores for production of energy especially in stress states like fasting (9). Hence, the entire study group was tested for the presence of non ketotic hypoglycemia, as a first sign of decreased rate of fatty acid oxidation.

Other markers like ammonia, lactate, transaminases, and CK levels, were measured as well to assess the degree of the affection of the different systems. 
The organic acid profile can explain one of the possible fates of metabolism of the accumulated free fatty acids (FFAs), which activate the $\omega$-oxidation pathway by which these FFAs are converted into dicarboxylic acids of chain lengths corresponding to the starting FFAs (12).

One of the common IEM is MCADD, its prevalence 1:17.000 live births (13). MCADD accounts for 1 of each SID syndrome (14). The majority of diagnosed patients with MCADD in this study had history of SIDS; similar findings were reported by Marci and Ajovalasit (15).

The main manifestation was hepatic dysfunction appeared as hepatomegaly and fatty liver. This hepatic affection is mainly caused by lipid infiltration of the liver, because of the accumulated FFAs, which are converted to triglycerides and stored in the liver (16).

Vomiting was an obvious sign in some MCAD-deficient patients. It is thought that this recurrent vomiting is caused by hyperammonemia and by the circulating FFAs (16).

The presence of mild ketonuria with the almost normal blood glucose level in MCADD-patients can be explained by the fact that the long chain acyl CoAs enter to $\beta$-oxidation many times depending on their chain length releasing acetyl CoA each time until it reaches the medium chain length at the site of the blockage. These released acetyl CoA molecules then enter into ketogenesis pathways (17). These results resemble those concluded by Hoflack and others (18) who diagnosed two cases with typical MCADD.

The ACP resulted from the defect in the MCAD enzyme (17) characterized by an elevation in medium chain ACs. A similar ACP was detected by Marci and Ajovalasit (15). The organic acid profile showed an increased medium chain length dicarboxylic acids. Their urine also contained acetoacetate and 3-hydroxybutyrate. Divary and others (19) recorded a similar organic acid profile of a case with MCADD.

Another diagnosed FAOD was LCHADD which has a relatively high prevalence among IEM; 1:75000 in German population (20).

The most prominent manifestation was the repeated attacks of rhabdomyolysis, which was caused by decreased energy supply to the muscles causing their weakness and atrophy by time, causing increase in CK level due to elevation of CK-MM isoenzyme of the muscles, this abnormal elevation is enough for diagnosis of rhabdomyolysis (21).

The second characteristic manifestation was the advanced retinopathy, which is not characteristic in the other FAODs. Pathogenesis mechanisms proposed to explain the chorioretinopathy of LCHAD and mitochondrial trifunctional protein (MTP) deficiencies include secondary deficiency of fatty acid docosahexaenoic acid (DHA), energy deprivation, and toxicity of accumulating intermediates. Several $\beta$-oxidation proteins, including MTP, are expressed in human ocular tissues, particularly in the retinal pigment epithelium (RPE) (20).

Elevated lactate in the case of LCHADD in this study is because 3-hydroxypalmitoyl CoA inhibits pyruvate dehydrogenase (PDH) enzyme, so in case of LCHADD, the pyruvate is converted to lactate by lactate dehydrogenase enzyme (LDH), instead of its conversion to acetyl CoA by PDH (22).

The urine contained high levels of 3-hydroxylong chain dicarboxylic acids. These findings are similar to those reported by Pones with his colleagues (23) who reported four cases with LCHADD of age range 9 months to 3 years.

In addition, the lack of awareness with MADD (glutaric academia type II (GA II)), the diversity in the clinical presentation and manifestations of this disorder is very confusing and misleading to the pediatricians and this is the main cause in delaying the diagnosis (24).

The manifestations accompanied with this disorder are most probably the result of defect in the catabolism of amino acids, not only fatty acids that negatively affect many systems in the body. The involved amino acids are valine, leucine, isoleucine, tryptophane, and lysine (25). This disorder is due to a defect or deficiency in any of the mitochondrial FAD-dependant dehydrogenases that are involved in fatty acid $\beta$-oxidation and many amino acids catabolism especially glutaric acid metabolism (25).

The ACP was very characteristic; it involved high amounts of most of AC species (short-, medium-, and long chain acylcarnitines). The organic acid profile was also very significant; it included enormous amounts of glutaric acid, ethylmalonic acid, 2-hydroxyglutaric acid, and dicarboxylic acids of variable chain lengths.

Different findings were reported by Wasant and others (25), when they reported a novel presentation of late onset GA II in two Thai siblings.

In the present study, the diagnosed patients group included some patients diagnosed previously with RI and they used to undergo hemodialysis therapy for long times. The ACP of these patients explains the effect of the renal diseases and subsequent chronic hemodialysis therapy on carnitine bioavailability to form the acylcarnitine esters (26). Before the hemodialysis, there was an abnormal increase in concentrations of both free carnitine and acetylcarnitine; which are normally present in high concentrations compared to the other ACs. They were elevated because of decreased clearance of these physiologic compounds as a result of RI. Another cause of SCD in these RI patients with is that the kidney is a major site for carnitine biosynthesis as the enzyme 6-N-trimethyllysine hydroxylase has activity in the kidneys four times in the other tissues; also the other enzymes involved in the carnitine biosynthesis have high activity in the kidneys (27). After termination of the hemodialysis; ACP indicated that there was a marked decrease in the free carnitine concentration because of the irreversible loss of the carnitine molecules through the dialysis membrane. There was a very small peak for free carnitine compared to its ISTD. In 2000, Evans and colleagues (28) conducted a similar study with 12 patients undergoing long-term hemodialysis, and they had results that agree with ours.

The SCD was predisposed in many patients who shared a common history of being on VPA therapy for long time in order to control seizures of different etiology. This relatively high number of patients is due to lack of awareness at many pediatricians who still prescribe this drug as an antiepileptic medication ignoring many researches that proved the fatal consequences of the administra- 
tion of this drug in cases of IEM disorders $(29,30)$. The SCD was produced from the consumption of free carnitine by VPA forming valproylcarnitine, which enters the mitochondria and undergoes $\beta$-oxidation producing propionyl-CoA and pentanoyl-CoA, which are then converted into propionylcarnitine and pentanoylcarnitine by the action of carnitine/acylcarnitine transtransferase (CAT) enzyme in the presence of free carnitine (31).

The main clinical manifestation was the hepatotoxicity, which is assumed to be caused by lipid peroxidation and glutathione reduction (32). The second manifestation observed was cardiotoxicity owing to the presence of high amounts of FFAs (33).

Hyperammonemia was obvious in these patients and this was supported by many researches which have proven that the most serious side effect of VPA administration was the aggressive hyperammonemia. There are many mechanisms suggesting the pathways of increasing ammonia in these patients. In the kidney; VPA increases the transport of glutamine across the mitochondrial membrane making it available for ammonia production. It also stimulates the glutaminase enzyme, thereby increases the conversion of glutamine to glutamate and release of ammonia. In the liver, it inhibits carbamoylphosphate synthetase I (CPS I), thereby preventing the incorporation of ammonia into the urea cycle; this inhibition is through the inhibition of $\mathrm{N}$-acetylglutamate; an activator of CPS I. It also increases pyruvate, which is a potent inhibitor of CPS I (34).

Together with the patients undergoing hemodialysis therapy, the valproate-administering patients with SCD had normal organic acid profile; this can be attributed to the fact that when free carnitine decreased, acyl CoAs accumulate then undergo peroxisomal $\beta$-oxidation producing medium chain acyl CoAs and dicarboxylic acids which don't require to bind to carnitine to enter the mitochondria and undergo complete oxidation (12).

The diagnosed patients included one case of GA I. This disorder results from the deficiency in FAD-dependent glutaryl CoA dehydrogenase enzyme causes accumulation of many intermediates of some amino acids catabolism (lysine, hydroxylysine, and tryptophane) in the basal ganglia leading to movement disorders (35).

Ketonuria was present despite SCD as medium- and short chain fatty acids undergo $\beta$-oxidation and produce acetyl CoA then ketogenesis as this patient has intact $\beta$-oxidation pathway (35). In this case, FAOD is considered to be a secondary metabolic disorder; because the overproduction of glutaric acid causes SCD as glutaric acid, like the other organic acids, is conjugated to free carnitine, and then excreted in urine as glutarylcarnitine. The ACP analysis revealed elevated amounts of $\mathrm{C}_{5} \mathrm{DC}$. Glutaric and 3-hydroxyglutaric acids were the most abundant organic acids in her urine due to decreased glutaric acid metabolism. These results agree with those of another case of GA I reported by Dong et al (35).

The major type of MMA results from deficiency in methylmalonyl CoA mutase, which converts methylmalonyl CoA, which derived from propionyl CoA to succinyl CoA, which enters the TCA cycle then converted to pyruvate. The other types of MMA are due to either defect in the intracellular cobalamine metabolism in the lysosoms, cytoplasm or mitochondria or due to deficiency in dietary cobalamine, or deficiency in transcobalamine (intrinsic factor) (36).

The complete picture of MMA was reported by Serinivas and others (37). ACP analysis showed elevated butyl-, propionyl-, and 3-hydroxybutylcarnitines, and decreased free carnitine. The organic acid profile revealed abnormally high methylmalonic acid. In addition to these findings, the diagnosis of MMA was confirmed by detecting the presence of elevated amounts of glycine and alanine in blood of the patient. Methylmalonic aciduria and ketotic hyperglycinemia coexist together and some times they are considered to be synonyms, the mechanism by which the glycine is elevated in urine and blood is still not determined (38).

In a previous study in Egypt on FAODs of 44 high risk children with a range of age similar to that of our study was performed by Hindawy et al (39); 16 patients were diagnosed with FAODs, 4 patients had MCADD, 3 had VLCADD, and 9 patients were diagnosed with MADD.

\section{Conclusion}

The ACP detection by LC/MS/MS is the key to diagnose many disorders presented as primary IEM or as a result of many metabolic or non metabolic conditions. This technique must be available in the developing countries, especially those with high consanguinity rates, to help in the early diagnosis and treatment of such cases.

Moreover, many of these disorders can be managed by simple measures like avoid fasting, avoid eating fat-rich meals, administration of adjusted dose of L-carnitine, avoid prescribing drugs that consume free carnitine (e.g. VPA), and finally, increase the sensibility to explain the serious consequences of consanguineous marriage. Another large-scale study is recommended to determine the incidence of fatty acid oxidation disorders among the Egyptian population.

\section{References}

1. Robert, Osprian, Tilg et al. Dynamic simulations on the mitochondrial fatty acid Beta-oxidation network. BMC Systems Biology 2009; (3): 3-2.

2. Kompare M, Rizzo W. Mitochondrial Fatty-Acid Oxidation Disorders. Clin Gene 2008; 15: 140-149.

3. Santra S, Hendriksz C. How to use acylcarnitine profiles to help diagnose inborn errors of metabolism. Arch Dis Child Educ Pract Ed 2010; $95(5)$.

4. Rinaldo, Piero, Cowan et al. Acylcarnitine profile analysis (ACMG Standards and Guidelines). Genet Med 2008; 10: 151-156.

5. Smith E, Matern D. Acylcarnitine Analysis by Tandem Mass Spectrometry. Curr Protoc Hum Genet 2010; 64: 17.8.1-17.8.20.

6. Scott R, Jamal A, Ibdaha BC. Fatty acid oxidation disorders: maternal health and neonatal outcomes. ELSEVIER, seminars in fetal\& neonatal 2010; 15 (3): 122-128.

7. Eric LK, Law M. Diagnosis of fatty acid oxidation disorders by mass spectrometry. Clin Chim Acta 2010; 411: 11-12. 
8. Pitt J, Egginton M, Kahler S. Comprehensive screening of urine samples for inborn errors of metabolism by electrospray tandem mass spectrometry. J Clin Chem 2002; 48: 1970-1980.

9. Bennett MJ. Pathophysiology of fatty acid oxidation disorders. Inher Metab Dis 2009; 42: 1134-1142.

10. Wilcken B. Fatty acid oxidation disorders: outcome and long-term prognosis. J Inherit Metab Dis 2010; 33 (5): 501-506.

11. Temtamy S, Afifi H, El- Ruby M et al.The most encountered groups of genetic disorders in Giza governorate, Egypt. Bratis Lek Listy 2012; 111 (2): 62-69.

12. Bruce A. Metabolomic approaches to mitochondrial disease: correlation of urine organic acids. Mitochondrion 2004; 4: 521-527.

13. Zytkovicz H, Comeau A, Strauss A et al. Spectrum of Medium-Chain Acyl-CoA Dehydrogenase Deficiency Detected by Newborn Screening. Ped 2008; 5: 1108-1114.

14. Russell LK, Bennett MJ, Tokunaga C. Reye-like syndrome resulting from novel missense mutations in mitochondrial medium- and short-chain L-3-hydroxy acyl-CoA dehydrogenase. Mol Genet Metab 2006; 89:74-79.

15. Marcì M, Ajovalasit P. Case Report: Medium-Chain Acyl-CoA Dehydrogenase Deficiency in an Infant with Dilated Cardiomyopathy. Cardiol Res Pract 2009.

16. Khalid JM, Dezateux C, Oerton J et al. The UK Collaborative Study of Newborn Screening for medium chain acyl CoA dehydrogenase deficiency (UKCSNS-MCADD): Overview and early findings. Arch Ped 2009; 17 (7): 1074-1077.

17. Yusuf $\mathbf{K}$, Jirapradittha $\mathbf{J}$, Amin $\mathbf{H J}$ et al. Neonatal Ventricular Tachyarrhythmias in Medium Chain Acyl-CoA Dehydrogenase Deficiency. Neonatology 2010; 98: 260-264.

18. Hoflack M, Caruba C, Pitelet G et al. Infant coma in emergency department: 2 cases of MCAD deficiency. Arch Pédiatr 2010; 7: 1074-1077.

19. Divry P, David M, Gregersen $\mathbf{N}$ et al. Dicarboxylic aciduria due to medium chain acyl-CoA dehydrogenase defect, A Cause of Hypoglycemia in Childhood. Acta Pediatr 2008; 72: 943-949.

20. Roomets E, Kivelä T, Tyni T. Carnitine Palmitoyl transferase I and Acyl-CoA Dehydrogenase 9 in Retina: Insights of Retinopathy in Mitochondrial Trifunctional Protein Defects. Invest Ophthalmol Visual Sci 2008; 49:1660-1664

21. Siqueira R, Bezerra G, Braga A, De Francesco A. Acute Kidney Injury due to Rhabdomyolysis. Saudi J Kidney Dis Transplant 2008; 5: 721-729.

22. Jason RB. Potential of gene therapy for the treatment of metabolic abnormalities in the heart. Int J Exp Pathol 2000; 6: 349-372.

23. Pones R, Roig M, Riudor $\mathbf{E}$ et al. The clinical spectrum of long-chain 3-hydroxyacyl-CoA dehydrogenase deficiency. Ped Neur 1996; 3: 236-243.
24. Mumtaz HA, Gupta V, Singh P et al. MR imaging findings of glutaric aciduria type II. Singapore Med J 2009; 4: 69-71.

25. Wasant P, Kuptanon C, Vattanavicharn $\mathbf{N}$ et al. Glutaric Aciduria Type 2, Late Onset Type in Thai Siblings with Myopathy. J Pediatr Neurol 2010; 4: 279-282.

26. Judith L, Simmson P, Vehige J. Role of carnitine in disease. Nutrition\& Metabolism 2010; 7: 7-30.

27. El-Hattab, Ayman W, Li F et al. Maternal systemic primary carnitine deficiency uncovered by newborn screening: Clinical, biochemical, and molecular aspects. Gene Med 2010; 1: 19-24.

28. Evans AM, Faull G, Fornasini EF et al. Pharmacokinetics of Lcarnitine in patients with end-stage renal disease undergoing long-term hemodialysis. Clin Pharmacol Ther 2000; 3: 238-249.

29. Philippe G, Larrey, Dominique et al. Drug-induced acute liver failure. Eur J Gastroenterol Hepatol 2005; 2: 141-143.

30. Berkeley N, Zucker S. Hyperammonemic Encephalopathy Caused by Carnitine Deficiency. Gen Intern Med 2008; 2: 210-213.

31. Silva M, Ruiter J, Overmars $\mathbf{H}$ et al. Complete $\beta$-oxidation of valproate: cleavage of 3-oxovalproyl-CoA by a mitochondrial 3-oxoacyl-CoA thiolase. Biochem J 2002; 362: 755-760.

32. Lheureux P, Penaloza A, Zahir S et al. Science review: Carnitine in the treatment of valproic acid-induced toxicity - what is the evidence? Crit Care 2005; 5: 431-440.

33. Kronn D, Narendra R, Dereddy et al. Defects in long chain fatty acid oxidation presenting as severe cardiomyopathy and cardiogenic shock in infancy. Cardiol Young 2009; 19: 540-542.

34. Mittal V, Muralee S, Tampi R Case Report: Valproic Acid-Induced Hyperammonemia in the Elderly: A Review of the Literature. Case Rep Med 2009; 5.

35. Dong Park J, Lim B, Kim K et al. Glutaric Aciduria Type 1 in Korea: Report of Two Novel Mutations. J Korean Med Sci 2010; 6: 957-960.

36. Quadros E, Lai S, Nakayama $Y$ et al. Positive newborn screen for methylmalonic aciduria identifies the first mutation in TCblR/CD320, the gene for cellular uptake of transcobalamine-bound vitamin $B_{12}$. Human Mutat 2010; 8: 924-929.

37. Serinivas KV, Want M, Freigoun $\mathrm{O}$ et al. Methylmalonic Acidemia with Renal Involvement: A Case Report and Review of Literature. Saudi J Kidney Dis Transplant 2010; 1: 49-53.

38. Bennett MJ. Untargeted Metabolomic Analysis Hits the Target. Clin Chem 2007; 53: 2037-2039.

39. Hindawy A, Gouda A, El-Ayyadi A et al. Metabolic encephalopathy in Egyptian children. Bratisl Lek Listy 2007; 2: 75-82.

Received April 30, 2012. Accepted February 28, 2014. 